\title{
IMPACT OF USING THE NEW THIRD FREQUENCY ON GPS AMBIGUITY RESOLUTION
}

\author{
Ali El-sharkawi ${ }^{1}$, Mohamed Abdel_hamid ${ }^{2}$, Reda Ali $^{3}$ \\ ${ }^{1}$ professor $\&^{2}$ Engineer ${ }^{3}{ }^{2}$ Lecturer, Faculty of Engineering, Helwan University, El-Mataria, Cairo, Egypt
}

\begin{abstract}
The new GPS L5 signal, is a part of the U.S. efforts to modernize its global positioning system (GPS). It was designed to support safety of life applications such as civil aviation navigation. Its structure designed to provide higher performance in terms of measurement accuracy. Till now, the GPS system cannot transmit L5 from all satellites. So, in this paper a GPS simulator software in MATLAB environment was implemented to simulate RINEX observations files with the three frequencies. MATLAB: GPSDP (GPS Differential Processing) program was used to process static and kinematic data from all three frequencies .

RINEX files for both base and rover stations had been generated for different baselines lengths 5,50 and $100 \mathrm{~km}$ approximately in different day times. These data are used to study the impact of the third frequency on rate of resolving integer ambiguities and the time needed to fix the integer ambiguities correctly. Also, the positioning accuracy after adding the third frequency to dual frequencies data. All parameters are studied under variation of ionosphere intensities.

The results of experiments have been illustrated and they show efficiency of using the third frequency over dual frequencies.
\end{abstract}




\section{INTRODUCTION}

The GPS satellite broadcast ranging codes and navigation data on two old frequencies named L1 (1575.42 MHZ), L2 (1227.6 MHZ) and new frequency L5 (1176 MHZ). Each satellite transmits on these old frequencies and some of them tell now transmit three frequencies L1, L2 and L5 but with different ranging codes than these employed by other satellites.

A major focus of the GPS modernization program is the addition of new navigation signals to the satellite constellation. [Governate, 2020]. The government is in the process of fielding three new signals designed for civilian use: L2C, L5, and L1C. The legacy civil signal, called L1 C/A or C/A at L1, will continue broadcasting, for a total of four civil GPS signals. Users must upgrade their equipment to benefit from the new signals.

The new civil signals are phasing in incrementally as the Air Force launches new GPS satellites to replace older ones. Most of the new signals will be of limited use until they are broadcast from 18 to 24 satellites. Once L2C and L5 are fully operational, their features will obviate the need for codeless or semi-codeless GPS receivers, which many GPS professionals use today to attain very high accuracy. Such receivers work by exploiting characteristics of the encrypted military $\mathrm{P}(\mathrm{Y})$ signal at the L2 frequency to achieve dual-frequency capability. [Governate, 2020]

L5 is the third civilian GPS signal, designed to meet demanding requirements for safety-of-life transportation and other high-performance applications. Its name refers to the U.S. designation for the radio frequency used by the signal (1176 $\mathrm{MHz}$ ). L5 is broadcast in a radio band reserved exclusively for aviation safety services. It features are higher power, greater bandwidth, and an advanced signal design. [Governate, 2020]

Future aircraft will use L5 in combination with L1 C/A to improve accuracy (via ionospheric correction) and robustness (via signal redundancy). In addition to enhancing safety, L5 use will increase capacity and fuel efficiency within U.S. airspace, railroads, waterways, and highways

This research is concern on the implementation of the third frequency L5 only on the ambiguity resolution behavior. A simulation program was constructed to simulate the GPS data of the three frequencies as the real data from all GPS satellites are not available yet. 


\section{GEOMETRY BASED CASCADING AMBIGUITY RESOLUTION}

The technique for ambiguity resolution using three carriers is denoted as a ThreeCarrier Ambiguity resolution (TCAR) and it could be classified in to two basic classifications, geometry free and geometry-based integer determination models, [Bofeng and Yunzhong, 2010]. There are many advantages when using triple frequency phase data because of the linear combinations that can be formed like Extra Wide Lane (EWL), and Wide Lane (WL) techniques. The frequency of are

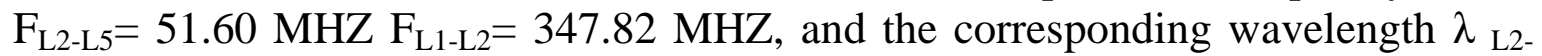
${ }_{\mathrm{L} 5} \approx 58.60 \mathrm{~cm}$ and $\lambda_{\mathrm{L} 1-\mathrm{L} 2} \approx 86.20 \mathrm{~cm}$ while the original wavelengths of L1, L2, and L5 were $19 \mathrm{~cm}, 24 \mathrm{~cm}$ and $25.5 \mathrm{~cm}$ respectively. The increased wide lane wavelength $\lambda_{\text {L2-L5 }}$ or $\lambda_{\text {L1-L2 }}$ provides an increased ambiguity spacing.

The cascading ambiguity resolution for GPS consists of three steps. Using the most precise code rang, the EWL ambiguities are resolved in first step, then the fixed EWL ranges are used to resolve the WL ambiguities, at the end the fixed WL ranges are used to resolve L1 ambiguities. [Feng, 2008]

In the geometry-based cascading ambiguity method, the geometric components are used. The general function model is illustrated in equation (1). [ Zhang, 2005]

$\mathrm{I}+\mathrm{r}=\mathrm{AX}$

$\mathrm{r} \quad$ is the observation foul

I is the observation vector

$\mathrm{X}$ is the state vector

A is the design matrix

\subsection{First step: Extra wide lane ambiguity resolution}

The observation vector consists of the Double Differenced DD EWL measurements and the DD of L5 code measurements:

$\mathrm{I}=\left[\begin{array}{c}\nabla \Delta F_{E W L} \\ \nabla \Delta_{3}\end{array}\right]$

$\nabla \Delta F_{E W L} \quad$ is the vector of DD EWL phase measurements.

$\nabla \Delta_{3} \quad$ is the vector of DD of the code measurements. 
The design matrix A:

$\boldsymbol{A}=\left[\begin{array}{cccccc}\frac{\partial \mathrm{f}^{\mathrm{i}}}{\partial \mathrm{x}} & \frac{\partial \mathrm{f}^{\mathrm{i}}}{\partial \mathrm{Y}} & \frac{\partial \mathrm{f}^{\mathrm{i}}}{\partial \mathrm{Z}} & \lambda_{E W L} & 0 & \ldots \\ \frac{\partial \mathrm{f}^{\mathrm{j}}}{\partial \mathrm{x}} & \frac{\partial \mathrm{f}^{\mathrm{j}}}{\partial \mathrm{Y}} & \frac{\partial \mathrm{f}^{\mathrm{j}}}{\partial \mathrm{Z}} & 0 & \lambda_{\text {EWL }} & \ldots \\ \cdots & \cdots & \ldots & \ldots & \ldots & \ldots \\ \frac{\partial \mathrm{f}^{\mathrm{i}}}{\partial \mathrm{x}} & \frac{\partial \mathrm{f}^{\mathrm{i}}}{\partial \mathrm{Y}} & \frac{\partial \mathrm{f}^{\mathrm{i}}}{\partial \mathrm{Z}} & 0 & 0 & \ldots \\ \frac{\partial \mathrm{f}^{\mathrm{j}}}{\partial \mathrm{x}} & \frac{\partial \mathrm{f}^{\mathrm{j}}}{\partial \mathrm{Y}} & \frac{\partial \mathrm{f}^{\mathrm{j}}}{\partial \mathrm{Z}} & 0 & 0 & \ldots \\ \ldots & \ldots & \ldots & \ldots & \ldots & \ldots\end{array}\right]$

Where:

$\frac{\partial \mathrm{f}^{\mathrm{i}}}{\partial \mathrm{x}}, \frac{\partial \mathrm{f}^{\mathrm{i}}}{\partial \mathrm{Y}}, \frac{\partial \mathrm{f}^{\mathrm{i}}}{\partial \mathrm{Z}}$ are partial derivate terms

$\mathrm{i}, \mathrm{j}$ are different satellites

$x=\left[\begin{array}{lllll}\delta x & \delta y & \delta Z & \delta\left(\nabla \Delta N_{L 1}\right) & \cdots\end{array}\right]^{T} \quad$ where $\quad \delta X=X-X_{0}, \delta Y=Y-Y_{0}, \delta Z=$

$Z-Z_{0}, \delta\left(\nabla \Delta N_{E W L}\right)=\nabla \Delta N_{E W L}-\nabla \Delta N_{E W L_{0}}$

$\mathrm{X}, \mathrm{Y}, \mathrm{Z} \quad$ are the final receiver position

$\mathrm{X} 0, \mathrm{Y} 0, \mathrm{Z} 0 \quad$ are the initial receiver position

$\nabla \Delta N_{E W L} \quad$ is the actual ambiguity

$\nabla \Delta N_{E W L_{0}} \quad$ is the initial ambiguity

0

means the initial or the pervious state

Float EWL ambiguities can be estimated through least square:

$Q_{x}=\left(A^{T} Q_{1}^{-1} A+Q_{x_{0}}^{-1}\right)^{-1}$

$X=-Q_{x} A^{T} Q_{1}^{-1}\left(1-A X_{0}\right)$

$Q_{1} \quad$ is the measurement variance covariance matrix

$Q_{x} \quad$ is the state variance matrix 
At the same time of estimate, the float ambiguities, the user position is updated. Then, the float EWL ambiguities on VC matrix are submitted to the ambiguity decorrelation and search algorithm (LAMBADA). [Teunissen, 2012]. If the EWL ambiguities cannot be fixed at the current epoch, then the processing proceeds to the next epoch till the EWL ambiguities are resolved then the algorithm goes to the second step.

\subsection{Second step: Wide Lane Ambiguity Resolution}

The observation vector consists of the DD fixed EWL ranges and DD WL phase measurements:

$\mathrm{I}=\left[\begin{array}{c}\nabla \Delta F_{W L} \\ \nabla \Delta F_{E W L}\end{array}\right]$

where:

$\nabla \Delta F_{E W L} \quad$ is the vector of the DD fixed EWL

$\nabla \Delta F_{W L} \quad$ is the vector of DD of WL phase measurements

The design matrix $\mathrm{A}$ is the same as that at the first step except changing $\lambda_{E W L}$ by $\lambda_{W L}$

The float WL ambiguities are first obtained from least square equation and then passed to the ambiguity searching after user position is updated. With fixed WL ranges, the cascading $\mathrm{AR}$ is translating to the third step.

\subsection{Third step: L1 Ambiguity Resolution}

The observation vector consists of raw three phases ranges and fixed DD EWL and DD WL ambiguity:

$\mathrm{I}=\left[\begin{array}{c}\nabla \Delta F_{L 1} \\ \left(\nabla \Delta F_{L 2}-\nabla \Delta N_{W l}\right) * \lambda_{2} \\ \left(\nabla \Delta F_{L 5}-\nabla \Delta N_{W l}-\nabla \Delta N_{E W L}\right) * \lambda_{5}\end{array}\right]$

Where $\nabla \Delta F_{L 1} \nabla \Delta F_{L 2}$ and $\nabla \Delta F_{L 5}$ are the vector of DD L1, L2 and L5 phase measurements respectively. 
The design matrix A:

$\boldsymbol{A}=\left[\begin{array}{cccccc}\frac{\partial \mathrm{f}^{\mathrm{i}}}{\partial \mathrm{x}} & \frac{\partial \mathrm{f}^{\mathrm{i}}}{\partial \mathrm{Y}} & \frac{\partial \mathrm{f}^{\mathrm{i}}}{\partial \mathrm{Z}} & \lambda_{\mathrm{L} 1} & 0 & \ldots \\ \frac{\partial \mathrm{f}^{\mathrm{j}}}{\partial \mathrm{x}} & \frac{\partial \mathrm{f}^{\mathrm{j}}}{\partial \mathrm{Y}} & \frac{\partial \mathrm{f}^{\mathrm{j}}}{\partial \mathrm{Z}} & 0 & \lambda_{\mathrm{L} 1} & \ldots \\ \cdots & \cdots & \ldots & \ldots & \ldots & \ldots \\ \frac{\partial \mathrm{f}^{\mathrm{i}}}{\partial \mathrm{x}} & \frac{\partial \mathrm{f}^{\mathrm{i}}}{\partial \mathrm{Y}} & \frac{\partial \mathrm{f}^{\mathrm{i}}}{\partial \mathrm{Z}} & \lambda_{\mathrm{L} 2} & 0 & \ldots \\ \frac{\partial \mathrm{f}}{\partial \mathrm{x}} & \frac{\partial \mathrm{f}^{\mathrm{j}}}{\partial \mathrm{Y}} & \frac{\partial \mathrm{f}^{\mathrm{j}}}{\partial \mathrm{Z}} & 0 & \lambda_{\mathrm{L} 2} & \ldots \\ \cdots & \ldots & \ldots & \ldots & \ldots & \ldots \\ \frac{\partial \mathrm{f}^{\mathrm{i}}}{\partial \mathrm{x}} & \frac{\partial \mathrm{f}^{\mathrm{i}}}{\partial \mathrm{Y}} & \frac{\partial \mathrm{f}^{\mathrm{i}}}{\partial \mathrm{Z}} & \lambda_{\mathrm{L} 5} & & \ldots \\ \frac{\partial \mathrm{f}^{\mathrm{j}}}{\partial \mathrm{x}} & \frac{\partial \mathrm{f}^{\mathrm{i}}}{\partial \mathrm{Y}} & \frac{\partial \mathrm{f}^{\mathrm{i}}}{\partial \mathrm{Z}} & & \lambda_{\mathrm{L} 5} & \ldots \\ \ldots & \ldots & \ldots & \ldots & \ldots & \ldots\end{array}\right]$

And the state vector is

$x=\left[\begin{array}{lllll}\delta x & \delta y & \delta z & \delta\left(\nabla \Delta N_{L 1}\right) & \cdots\end{array}\right]^{T} \quad$ where $\delta X=X-X_{0}, \delta Y=Y-Y_{0}, \delta Z=$ $Z-Z_{0}, \delta\left(\nabla \Delta N_{L 1}\right)=\nabla \Delta N_{L 1}-\nabla \Delta N_{L 1_{0}}$

all parameters are illustrated previously

The initial L1 ambiguities are estimated from least square equation Once the L1, WL and EWL ambiguities are fixed. It used to derive L2 and L5 ambiguities. Interested reader is advised to referee to [ Zhang, 2005] for more information.

An educational software GPS Differential Positioning (GPSDP) implements TCAR techniques as mentioned above to process GPS data for dual and triple frequencies. [Ali 2020].

\section{GPS SIMULATION DATA}

However, not all GPS satellites (13 out of 32) had the last update of new signals. [US Governate, 2020]. A GPS simulation program in MATLAB environments was developed to generate three frequency data for this investigation. Figure (1) illustrates the flowchart diagram and main user interactive windows of the program. The output from every station is the observation file including both header and 
observation data in RINEX version 2.10. [Gurtner, 2002] these data could be handled by any commercial or academic software. [AbdelHamid, 2020].
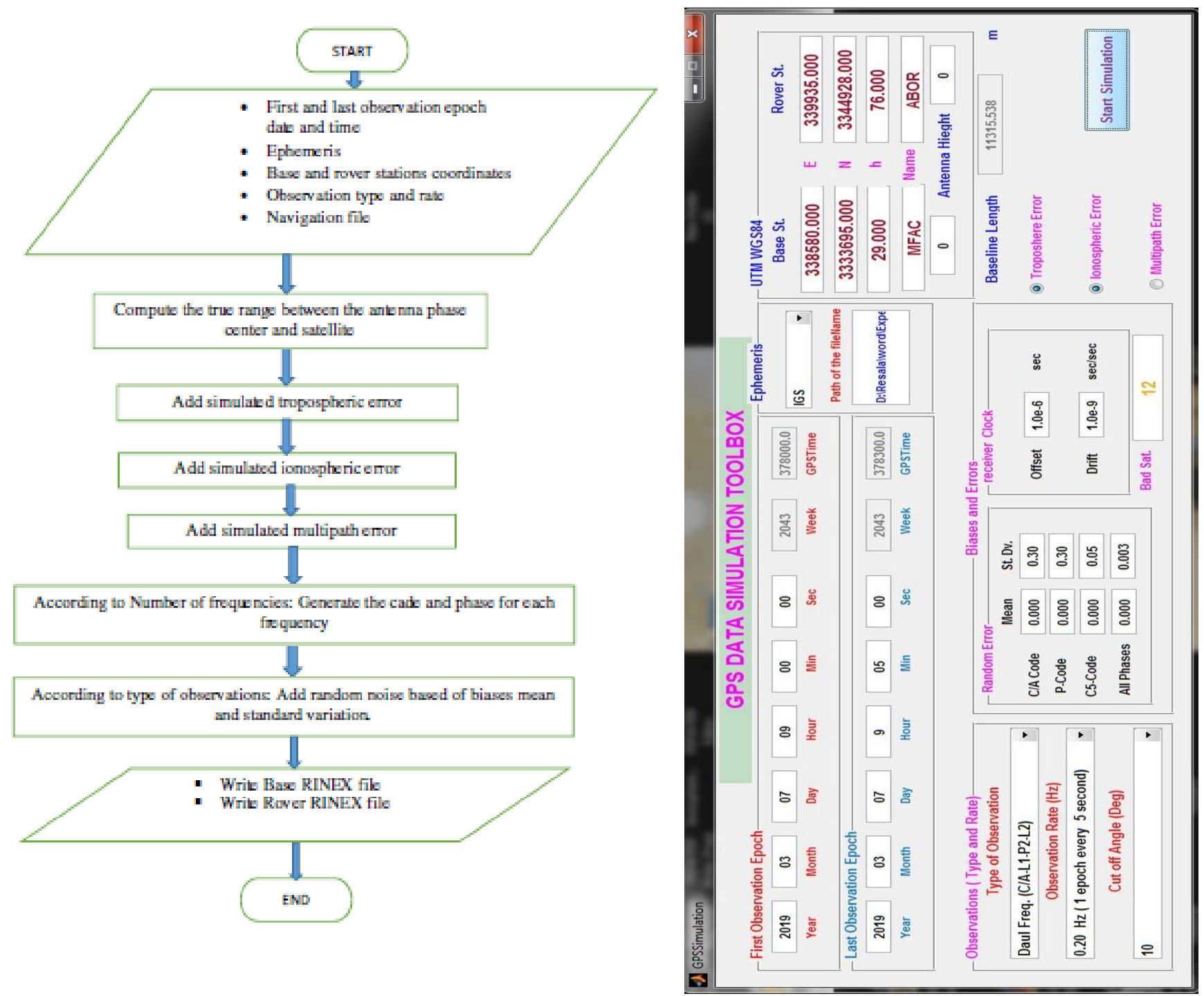

Figure (1) GPS Data Simulation Program

\section{EXPERIMENTS AND RESULTS}

Simulated RINEX files over 5, 50 and $100 \mathrm{~km}$ approximately baselines for different times are studied in this investigation. The coordinates of stations were listed in table (1). Where MFAC is chosen as the base station. all experiments had been run with dual frequencies $(\mathrm{L} 1+\mathrm{L} 2)$ and triple frequencies $(\mathrm{L} 1+\mathrm{L} 2+\mathrm{L} 5)$ respectively to study: 
- The time needed to fix the float L1 ambiguities to correct integer number when using (L1+L2) only and using (L1+L2+L5).

- The Ambiguity success rate which is the ratio between the number of epochs that the ambiguity had been solved correctly to the total number of epochs. when using (L1+L2) only and using (L1+L2+L5).

All these parameters are studied in different conditions of ionosphere error. GPS differential positioning program (GPSDP), it is a toolbox in MATLAB used to fix the ambiguity and measure the required time for fixing it,

Table (1) The UTM coordinates of stations used in simulation (in meters)

\begin{tabular}{|l|l|l|l|l|}
\hline Station & East Coord. & North Coord. & $\begin{array}{c}\text { Ellipsoidal } \\
\text { Height }\end{array}$ & \multicolumn{1}{c|}{ Remarks } \\
\hline MFAC & 338596.000 & 3333690.000 & 045.000 & Base Station \\
\hline A & 343462.000 & 3334771.000 & 031.000 & MFAC-A = 4985.117 m \\
\hline B & 389143.000 & 3329865.000 & 225.000 & MFAC-B = 50701.330 \\
\hline C & 348415.000 & 3433560.000 & 008.000 & MFAC-C \\
\hline
\end{tabular}

\subsection{Time to Fix L1 Ambiguity}

Data has been simulated in the first experiment carried out from 02:00 to 04:00 in local time where the ionosphere effect was low, the results showed that the time to fix the ambiguity minimized when we had turned from dual to triple frequency. the same happened in experiment two but the ionosphere effect was medium from 05:00 to 07:00 in local time. fig (2) showed the results from experiments 1 , and 2 . it is shown that, in short base lines no need to the new frequency in all cases of ionosphere but in medium baselines the time to fix the ambiguity minimized when we had translated from dual to triple frequency and in long baselines the same results in addition to that no solution in high ionosphere unless the three frequencies are used. 
Table (2) shows the required time to fix the ambiguity

\begin{tabular}{|c|c|c|c|c|}
\hline \multirow{2}{*}{$\begin{array}{c}\text { Base line } \\
(\mathrm{km})\end{array}$} & \multicolumn{2}{|c|}{$\begin{array}{c}\text { Time to fix the ambiguity L1 } \\
+\mathrm{L} 2(\mathrm{Sec})\end{array}$} & \multicolumn{2}{c|}{$\begin{array}{c}\text { Time to fix the ambiguity } \\
(\mathrm{L} 1+\mathrm{L} 2+\mathrm{L})(\mathrm{Sec})\end{array}$} \\
\cline { 2 - 5 } & EXP.1 & EXP.2 & EXP.1 & EXP.2 \\
\hline 5 & 3.3 & 2.5 & 3.3 & 2.5 \\
\hline 50 & 36.6 & 50 & 3.3 & 2.5 \\
\hline 100 & 100 & 90 & 3.3 & 5 \\
\hline
\end{tabular}

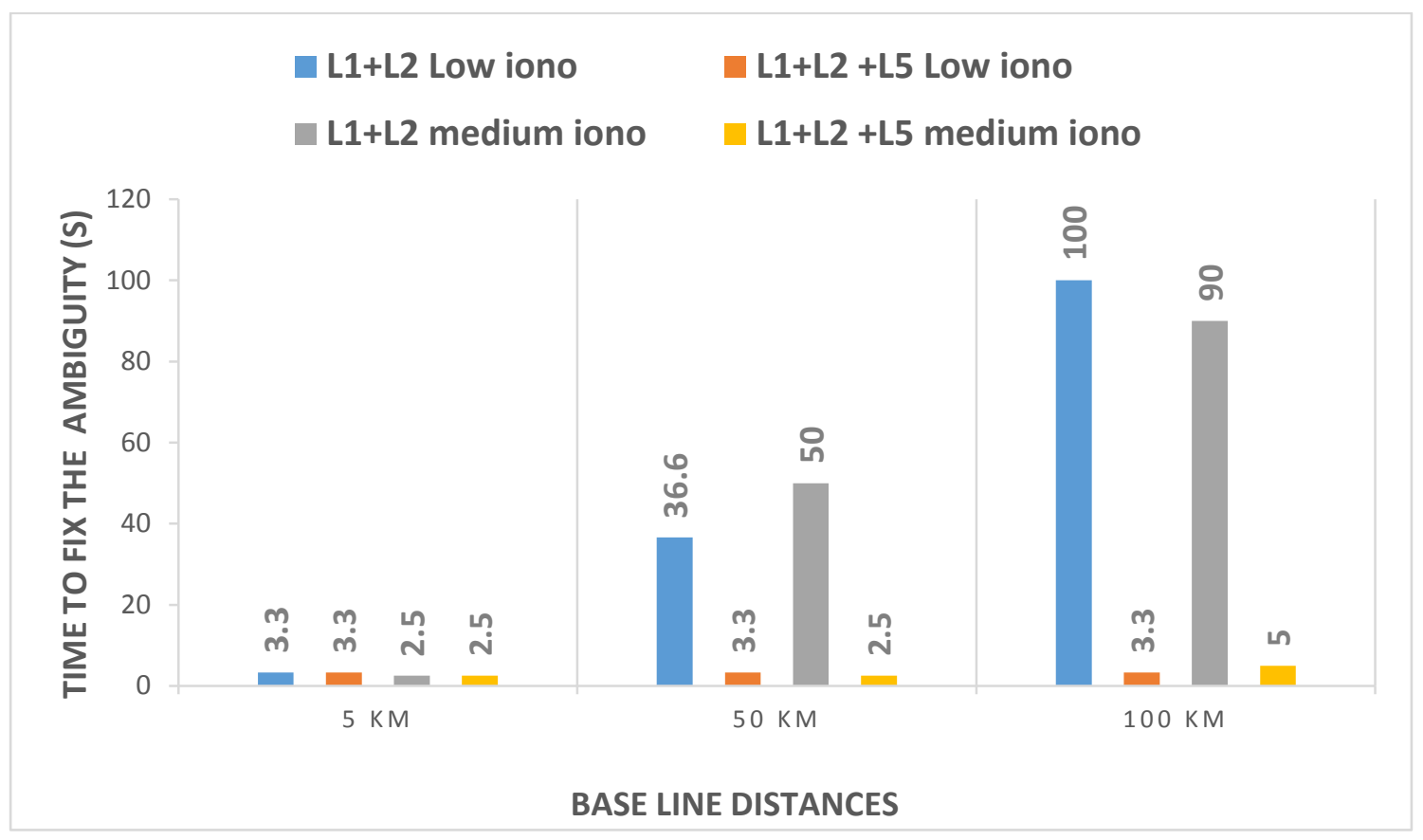

Fig (2) Time to fix the ambiguity in different ionosphere cases

\subsection{Ambiguity Success Rate}

The precise GPS application usually needs to fix the phase ambiguity, that takes the different time depending on application and observation scenarios. In general, the less time cost for ambiguity resolution, the earlier the user can achieve the precise positioning and then a higher availability. In real applications, one cannot always fix all ambiguities due to their different precisions and one does not necessarily to fix all ambiguities in sense of precision demand. [Bofeng, 2017]

Using data illustrated in previous section, the ambiguity success rate is computed for every observation scenario. The results are organized in table (3). 
It is obvious from the table that, wide lane ambiguity fixing rate was improved when using the third frequency, and it reached $99 \% \mathrm{f}$ or $100 \mathrm{~km}$ baseline length. This means that a promising accuracy could be obtained by adding of the third frequency.

Table (3) Ambiguity success rates in experiments (1-2)

\begin{tabular}{|c|c|c|c|c|c|c|}
\hline Baseline Distance & \multicolumn{2}{|c|}{$5 \mathrm{~km}$} & \multicolumn{2}{c|}{$50 \mathrm{~km}$} & \multicolumn{2}{c|}{$100 \mathrm{~km}$} \\
\hline $\begin{array}{c}\text { Ambiguity } \\
\text { Resolution }\end{array}$ & $\begin{array}{c}\text { Wide } \\
\text { Lane } \%\end{array}$ & L1\% & $\begin{array}{c}\text { Wide } \\
\text { Lane } \%\end{array}$ & L1\% & $\begin{array}{c}\text { Wide } \\
\text { Lane } \%\end{array}$ & L1\% \\
\hline \multicolumn{7}{|c|}{ Experiment no 1 } \\
\hline Dual Frequencies & 98.5 & 98.4 & 27.78 & 27.76 & 2.91 & 2 \\
\hline Triple Frequencies & 100 & 93.27 & 100 & 56.97 & 100 & 54.55 \\
\hline \multicolumn{7}{|c|}{ Experiment no 2 } \\
\hline Dual Frequencies & 92.3 & 92.3 & 6.62 & 11.91 & 6.38 & 3.68 \\
\hline Triple Frequencies & 100 & 94.1 & 100 & 48.23 & 99.79 & 41.71 \\
\hline
\end{tabular}

\section{CONCLUSION}

In this investigation the impact of adding the new third GPS frequency had studied on 3 baselines 5, 50 and $100 \mathrm{~km}$ approximately. The study showed that new third L5 frequency minimized the time to fix the ambiguity in case of low or medium ionosphere by factor of $\left(\frac{1}{3.50}\right)$. results also show the ease and solve for wide lane ambiguity and increase the success rate of ambiguity resolution of L1 specially in long baselines. These results enable the use of kinematic application of GPS over long distances more reliable. 


\section{REFERENCES}

1. Ali, Reda “ MATLAB: GPS Differential positioning Toolbox "GPS solution (under revision 2020).

2. Abdel_hamid M. A.' Impact of using the new third frequency on GPS positioning accuracy " MSC thesis, Faculty of Engineering Mattria, Cairo, Helwan university, Egypt. (2020).

3. Governate, USA. Official U.S. government information about the Global Positioning System (GPS) and related topics. 62020 . <https://www.gps.gov/>.

4. Gurtner, W. "RINEX: The Receiver Independent Exchange Format Version 2.10, ftp." igscb. jpl. nasa. gov/igscb/data/format (2002).

5. Feng, Yanming. "GNSS three carrier ambiguity resolution using ionospherereduced virtual signals." Journal of Geodesy 82.12 (2008): 847-862.

6. Li, Bofeng "ERTK: extra-wide-lane RTK of triple-frequency GNSS signals." Journal of geodesy 91.9 (2017): 1031-1047.

7. Li, Bofeng. "Review of triple-frequency GNSS: ambiguity resolution, benefits and challenges." The Journal of Global Positioning Systems 16.1 (2018).

8. Teunissen, Peter JG, and Alfred Kleusberg, eds. GPS for Geodesy. Springer Science \& Business Media, 2012.

9. Zhang, Wentao. Triple frequency cascading ambiguity resolution for modernized GPS and GALILEO. University of Calgary, Department of Geomatics Engineering, 2005. 